\title{
Zero-Resistance States Induced by Electromagnetic-Wave Excitation in GaAs/AlGaAs Heterostructures
}

\author{
R. G. Mani ${ }^{1,2, *}$ \\ ${ }^{1}$ Harvard University, Gordon McKay Laboratory of Applied Science, 9 Oxford Street, Cambridge, MA 02138, USA \\ ${ }^{2}$ Max-Planck-Institut für Festkörperforschung, Heisenbergstrasse 1, Stuttgart 70569, Germany
}

(Dated: June 15, 2003)

\begin{abstract}
We report the detection of novel zero-resistance states induced by electromagnetic wave excitation in ultra high mobility GaAs/AlGaAs heterostructure devices, at low magnetic fields, $B$, in the large filling factor limit. Vanishing resistance is observed in the vicinity of $B=[4 /(4 j+1)] B_{f}$, where $B_{f}=2 \pi \mathrm{fm}^{*} / e$, where $\mathrm{m}^{*}$ is the effective mass, e is the charge, and $f$ is the microwave frequency. The dependence of the effect is reported as a function of $f$, the temperature, and the power.
\end{abstract}

PACS numbers: 73.21.-b,73.40.-c,73.43.-f; Journal-Reference: Physica E (Amsterdam) 22, 1 (2004).

Vanishing electrical resistance in condensed matter has introduced new physical phenomena such as superconductivity, which developed from the detection of a zeroresistance state in a metal.[1] More recently, the discovery of quantum Hall effects (QHE) stemmed from studies of zero-resistance states at low temperatures $(T)$ and high magnetic fields $(B)$ in the 2-Dimensional Electron System (2DES).[2, 3, 4] Quantum Hall effect and superconductivity have shown that a complex electronic system can exhibit instantly-recognizable physical phenomena. They have also demonstrated that observations of vanishing resistance in unusual settings can be a harbinger of new physics. Here, we report the observation of novel vanishing resistance states in an unexpected setting the ultra high mobility 2DES irradiated by low energy photons, at low temperatures, in the low magnetic field, large filling factor limit. We find that GaAs/AlGaAs heterostructures including a 2DES exhibit vanishing diagonal resistance about $B=(4 / 5) B_{f}$ and $B=(4 / 9) B_{f}$, where $B_{f}=2 \pi f m^{*} / e, m^{*}$ is an effective mass, $e$ is the electron charge, and $f$ is the radiation frequency. And, the resistance-minima follow a series $B=[4 /(4 j+1)] B_{f}$ with $j=1,2,3 \ldots$ Remarkably, in this instance, vanishing resistance in the 2DES does not produce plateaus in the Hall resistance, although the diagonal resistance exhibits activated transport and zero-resistance states, similar to QHE. [5, 6]

Measurements were performed on Hall bars, square shaped devices, and Corbino rings, fabricated from GaAs/AlGaAs heterostructures. After a brief illumination by a red LED, the best material was typically characterized by an electron density, $n(4.2 \mathrm{~K}) \approx 3 \times 10^{11}$ $\mathrm{cm}^{-2}$, and an electron mobility $\mu(1.5 \mathrm{~K}) \approx 1.5 \times 10^{7}$ $\mathrm{cm}^{2} /$ Vs. Lock-in based four-terminal electrical measurements were carried out with the sample mounted inside a waveguide and immersed in pumped liquid He-3 or He4. Electromagnetic (EM) waves in the microwave part of the spectrum, $27 \leq f \leq 170 \mathrm{GHz}$, were generated using various tunable sources. The power level in the vicinity of the sample was typically $\leq 1 \mathrm{~mW}$. In this report, we shall mainly exhibit data which illustrate the characteristics of the phenomena. Expanded discussions appear elsewhere.[6, 7, 8, 9]

Fig. 1 (a) shows measurements of the diagonal $\left(R_{x x}\right)$ and Hall $\left(R_{x y}\right)$ resistances to $B=6$ Tesla where, under microwave excitation at $50 \mathrm{GHz}, R_{x x}$ and $R_{x y}$ exhibit the usual quantum Hall behavior for $B \geq 0.3$
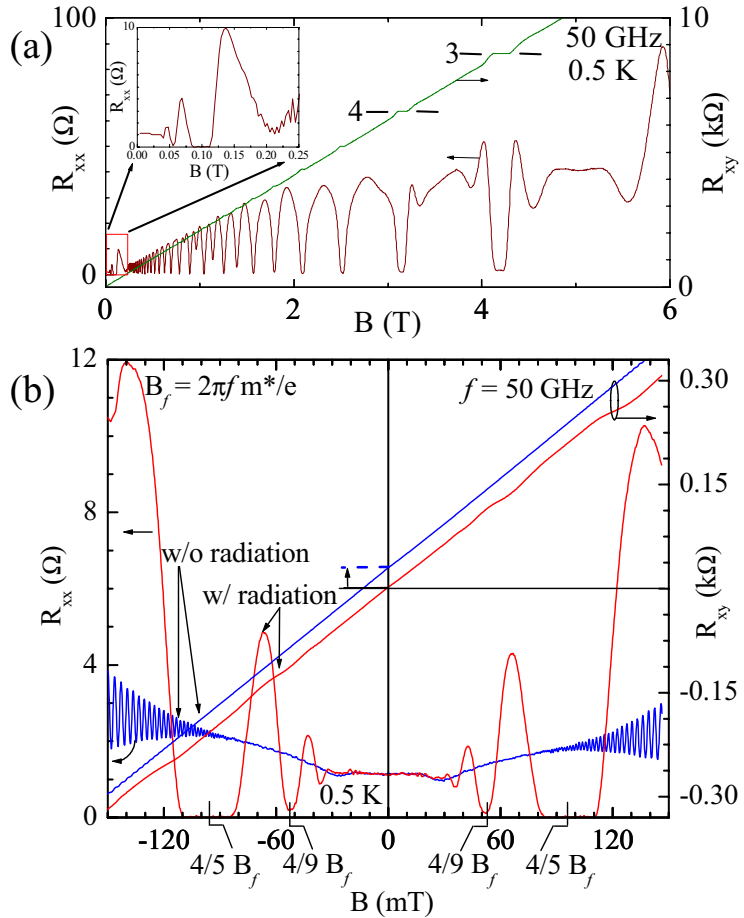

FIG. 1: (a): The Hall $\left(R_{x y}\right)$ and diagonal $\left(R_{x x}\right)$ resistances in a GaAs/AlGaAs heterostructure with excitation at $50 \mathrm{GHz}$. Quantum Hall effects (QHE) occur at high B as $R_{x x}$ vanishes. Inset: An expanded view of the low-B data. (b): Data over low magnetic fields obtained both with (w/) and without (w/o) excitation at $50 \mathrm{GHz}$. Here, radiation induced vanishing resistance about $(4 / 5) B_{f}$ does not induce plateaus in the Hall resistance, unlike in QHE. Yet, there are perceptible microwave induced oscillations in the Hall effect. 


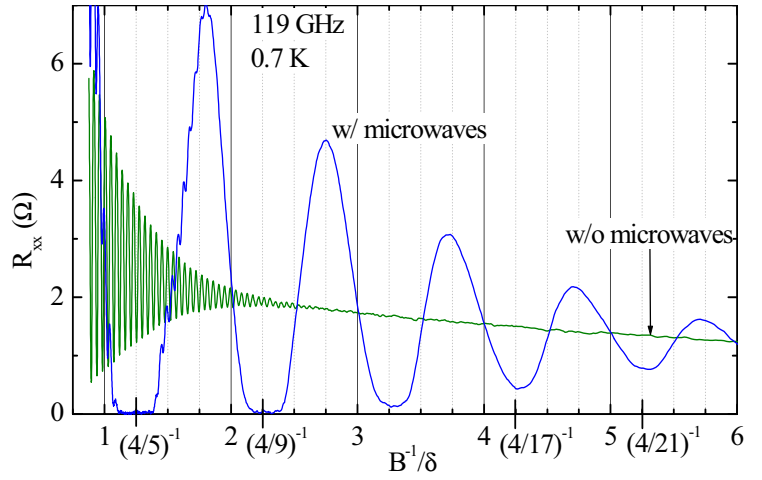

FIG. 2: A plot of $R_{x x}$ versus the normalized inverse magnetic field, $B^{-1} / \delta$, demonstrates periodicity of the radiation induced oscillations. The curves with- and without- radiation intersect near integral and half-integral values of the abscissa.

Tesla.[2, 3] In contrast, for $B<0.25$ Tesla, see inset of Fig. 1(a), a radiation induced signal occurs and the resistance vanishes over a broad B-interval about $B$ $=0.1$ Tesla. Further high-resolution measurements are shown in Fig. 1 (b). Without EM-excitation, $R_{x x}$ exhibits Shubnikov-deHaas oscillations for $B>100$ milliTesla (Fig. 1 (b)). The application of microwaves induces resistance oscillations, $[5,10,11]$ the resistance under radiation falls below the resistance without radiation, over broad $B$-intervals.[5, 8] Indeed, $R_{x x}$ appears to vanish about $(4 / 5) B_{f} .[6,7,9]$ Although these zeroresistance-states exhibit a flat bottom as in the quantum Hall regime,[2] $R_{x y}$ under radiation does not exhibit plateaus over the same $B$-interval.

As an example, Fig. 2 shows a normalized $B^{-1}$ plot of the response obtained under microwave excitation at $119 \mathrm{GHz}$. This plot shows that (i) the magnetoresistance oscillations are periodic in $B^{-1}$, (ii) the minima form about $B_{\text {min }}^{-1} / \delta=[4 /(4 j+1)]^{-1}$ with $j=1,2,3 \ldots$ Here, $\delta$ is the oscillatory period in $B^{-1}$, which is consistent with $B_{f}^{-1}$ within experimental uncertainty, (iii) the higher order maxima coincide with $B_{\max }^{-1} / \delta=[4 /(4 j+3)]^{-1}$. Experiment indicates that the resistance maxima generally obey this rule for integral $j$, excepting $j=0$, where phase distortion associated with the last peak seems to shift it from the $B_{\max }^{-1} / \delta=3 / 4$ to approximately $0.85( \pm 0.02)$ (see also Fig. 5), and (iv) the data obtained with radiation cross the data obtained without radiation, at integral and half-integral values of the $B^{-1} / \delta$, when $B^{-1} / \delta \geq 2$. At these $B^{-1} / \delta$, the photon energy $h f$ spans, perhaps, an integral, $j$, or half integral, $j+1 / 2$, cyclotron energies. The crossing feature near integral $B^{-1} / \delta$ looks to be in agreement with theory, $[12,13]$ which suggests that, when $2 \pi f / \omega_{C}=j$, the conductivity in the presence of radiation, $\sigma$, equals the conductivity in the absence of radiation, $\sigma_{d a r k}$, i.e., $\sigma=\sigma_{\text {dark. }}$.[13] Remarkably, the data

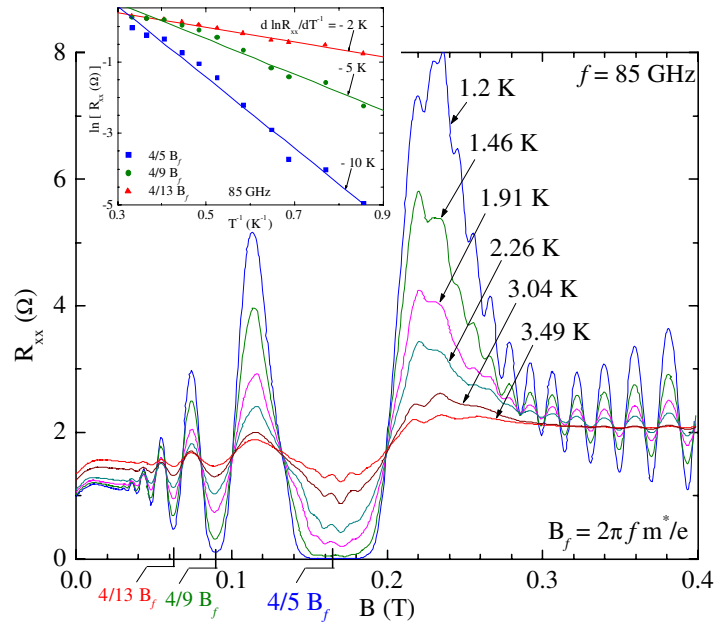

FIG. 3: The T-dependence of $R_{x x}$ at $85 \mathrm{GHz}$ under constantpower radiation. The radiation induced resistance minima become deeper at lower temperatures. (inset) A $\ln R_{x x}$ vs. $T^{-1}$ plot at $B=(4 / 5) B_{f},(4 / 9) B_{f}$, and $(4 / 13) B_{f}$ suggests activated transport. The activation energy $T_{0} \approx 10 \mathrm{~K}$ for the $(4 / 5) B_{f}$ state at $85 \mathrm{GHz}$.

suggest the same behavior at $j+1 / 2$.

The temperature variation of $R_{x x}$ at $85 \mathrm{GHz}$, shown in Fig. 3, displays both the strong $T$-dependence of $R_{x x}$ and the low- $T$ requirement for the observation of the radiation induced zero-resistance state. The T-variation of $R_{x x}$ at the deepest minima suggests activated transport, i.e., $R_{x x} \sim \exp \left(-T_{0} / T\right),[2,6]$ see inset Fig. 3 , and, in temperature units, the activation energy at $B=(4 / 5)$ $B_{f}, T_{0} \approx 10 \mathrm{~K}$ at $85 \mathrm{GHz}$, exceeds the Landau level spacing, $3.26 \mathrm{~K}$, and the photon energy, $h f=4.08 \mathrm{~K}$. Remarkably, $T_{0}$ is reduced as one moves to higher order minima at lower $B$, see inset Fig. 3. Further studies indicated that $T_{0}$ varied with the radiation intensity, as illustrated in Fig. 4.

These power dependent activation results go together with other measurements, which showed that the amplitude of the radiation induced resistance oscillations increased with the radiation intensity (see Fig. 3a,ref[6]), up to a (sample-dependent) optimum value of the radiation intensity (see Fig. 5). Concomitantly, the resistance in the vicinity of $B=[4 /(4 j+1)] B_{f}$ decreased and, about $(4 / 5) B_{f}$ and $(4 / 9) B_{f}, R_{x x} \rightarrow 0$. Current dependence measurements also demonstrated insensitivity to the magnitude of the current and the Hall electric field. $[6,7]$

The effect of tilting the specimen with respect the applied $B$, as the microwave radiation is directed along the $B$-axis, is illustrated in Fig. 6. Note that the field scale shown on the abscissa is different, for each panel in the figure. These results suggest that the radiation induced magnetoresistance, ignoring possible role for spin, 


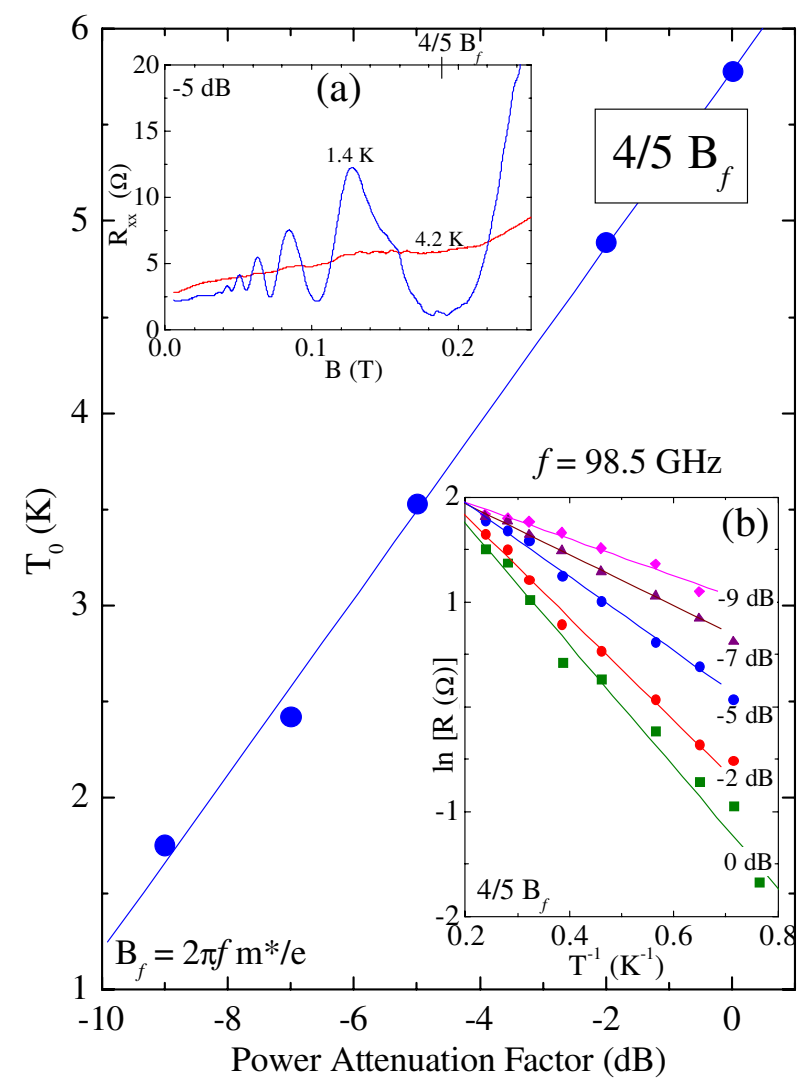

FIG. 4: The variation of the activation energy, $T_{0}$, as a function of the microwave power at $98.5 \mathrm{GHz}$ and $(4 / 5) B_{f}$. (a) $R_{x x}$ vs. $B$ at an attenuation factor of $-5 \mathrm{~dB}$. (b) The natural logarithm of $R_{x x}$ is shown versus the inverse temperature.

is mostly sensitive to the perpendicular component of the total magnetic field, $B_{\perp}=B \cos (\theta)$.

The orientation of the microwave polarization with respect to the current axis could be an important factor in experiment [14] and, therefore, results examining the role of this parameter are shown in Fig. 7. Measurements carried out at $39 \mathrm{GHz}$ on a sample mounted inside a WR-28 waveguide (see Fig. 7), with a current applied between the ends of the L-shaped device, suggest the same period and phase in the $E \| I$ and the $E \perp I$ configurations.

Some results of an investigation examining the $I$ dependence of the resistance in the Corbino geometry are exhibited in Fig. 8. This figure shows that the Corbino resistance is independent of the current, at relatively low currents, which supplements previously reported results for the Hall geometry (see Fig. 3(b), ref. [6]).

In this configuration, a maximum in the resistance (or a minimum in the conductance) occurs about $B=(4 / 5)$ $B_{f}$ and $B=(4 / 9) B_{f}$, in place of the resistance minimum observed in the Hall configuration (see Fig. 1). This geometry difference originates from a well-known feature of transport, that the resistance in the Corbino geometry $R_{C} \approx \sigma_{x x}^{-1}$, while in the Hall geometry, $R_{x x} \approx \sigma_{x x} / \sigma_{x y}^{2}$,

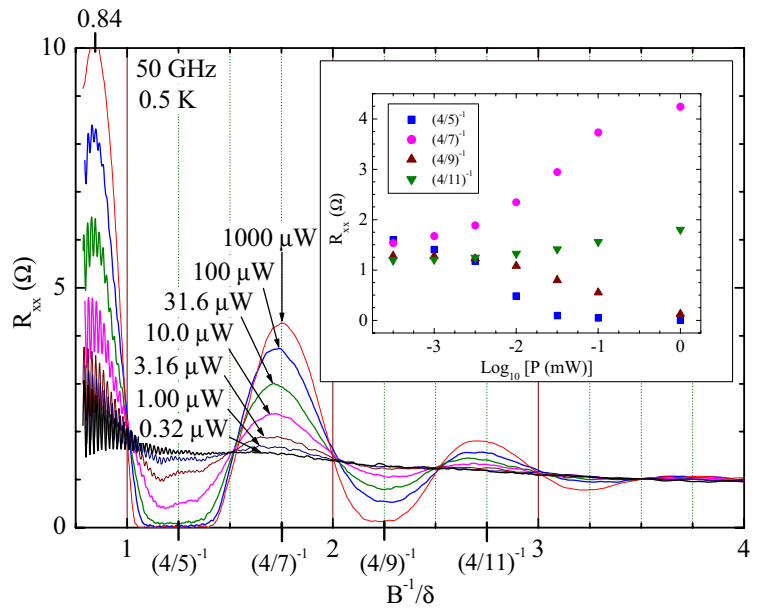

FIG. 5: Radiation induced resistance oscillations at $f=$ $50 \mathrm{GHz}$ are exhibited for various source intensities, in units of $\mu \mathrm{W}$. The inset shows the extrema resistance at $(4 / 5)^{-1}$, $(4 / 7)^{-1},(4 / 9)^{-1},(4 / 11)^{-1}$ vs. the logarithm of the power.

under $\omega_{C} \tau>1$ condition.

Preliminary results of experiments examining possible transmission and absorption characteristics under microwave excitation are illustrated in Fig. 9 and Fig. 10. For the transmission experiments (Fig. 9), a carbon resistor was placed immediately below the sample, and its resistance was measured simultaneously along with the specimen characteristics, as radiation was applied from above (see Fig. 9, inset). The magnetoresistance of the carbon resistor is shown in Fig. 9(a), while Fig. 9(b) illustrates $R_{x x}$ of the $2 \mathrm{DES}$ vs. $B$. Here, $-60 \mathrm{~dB}$ corresponds to vanishing excitation, while $0 \mathrm{~dB}$ corresponds to the maximum microwave intensity. In this sample, the optimum radiation induced $R_{x x}$ response, shown in Fig. 9 (b), was observed in the vicinity of $-8 \mathrm{~dB}$. That is, the amplitude of the radiation induced oscillations increased monotonically with increasing power attenuation factor up to $-8 \mathrm{~dB}$.

A further increase of the radiation intensity $(\mathrm{dB} \rightarrow 0)$ produced a reduction in the peak height along with an increase in the resistance at the minima (see Fig. 9(b)). This behavior suggests a "breakdown" of the radiation induced zero-resistance states at high excitation levels, which is somewhat analogous to the "breakdown" of the quantum Hall effect that is observed at high currents.[2] At the same time, the response of the carbon resistor placed below the sample (see Fig. 9(a)) suggests structure at magnetic fields mostly above $B_{f}$, which becomes more pronounced with increased excitation. The feature correlates with a strong radiation-induced decrease in $R_{x x}$ just above 0.3 Tesla. Observed oscillations in $R_{x x}$ below $B_{f}$ (Fig. 9(b)) are imperceptible in the detector response (see Fig. 9(a)). 

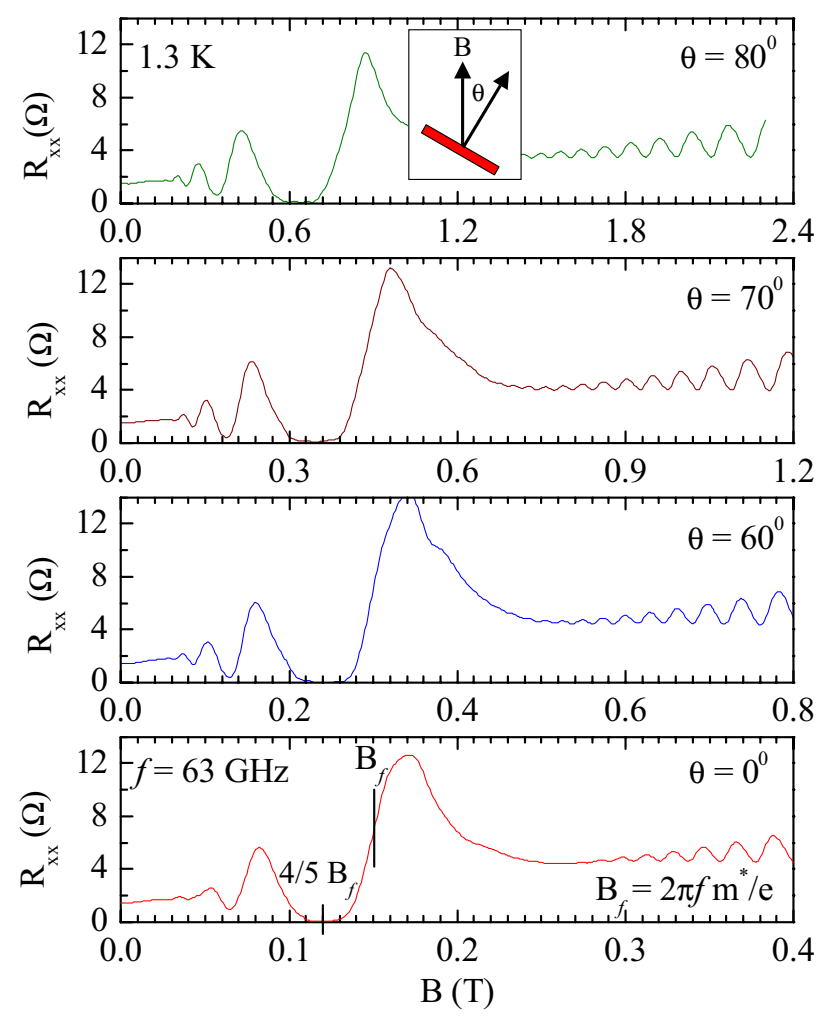

FIG. 6: Tilt-field measurements show that the radiation induced magnetoresistance is sensitive to the perpendicular component of total applied magnetic field.

The results of a measurement reflecting possible absorption characteristics of the specimen are illustrated in Fig. 10. In this experiment, the sample was mounted inside a waveguide that was susceptible to He-4 thermoacoustic oscillations (TAO).[15]

In this setup, the onset of TAO's under microwave excitation generated mechanical vibrations of the sample and thereby produced electrical noise in the measurement. However, this behavior was only observed for $B<$ $B_{f}$, which suggested the conjecture that energy absorption by the sample at $B<B_{f}$ modified the immediate sample environment and triggered the TAO's. That is, in Fig. 10, the 'noisy' portion of the data could signify absorption by the sample below $B_{f}$ (at $4.2 \mathrm{~K}$ ).

Radiation induced zero-resistance states have been confirmed in GaAs/AlGaAs quantum wells by Zudov et al.,[16], who suggested the effect as evidence of a new dissipationless effect in 2D electronic transport.

Phillips has suggested the effect to be a manifestation of sliding charge density waves.[17] Durst and co-workers have identified radiation induced resistance oscillations with a driven current, similar to Ryzhii.[12, 18]

Andreev et al.,[19] and Anderson and Brinkman,[20] suggested a physical instability for the negative resistivity state, while Andreev et al. proposed a scenario for

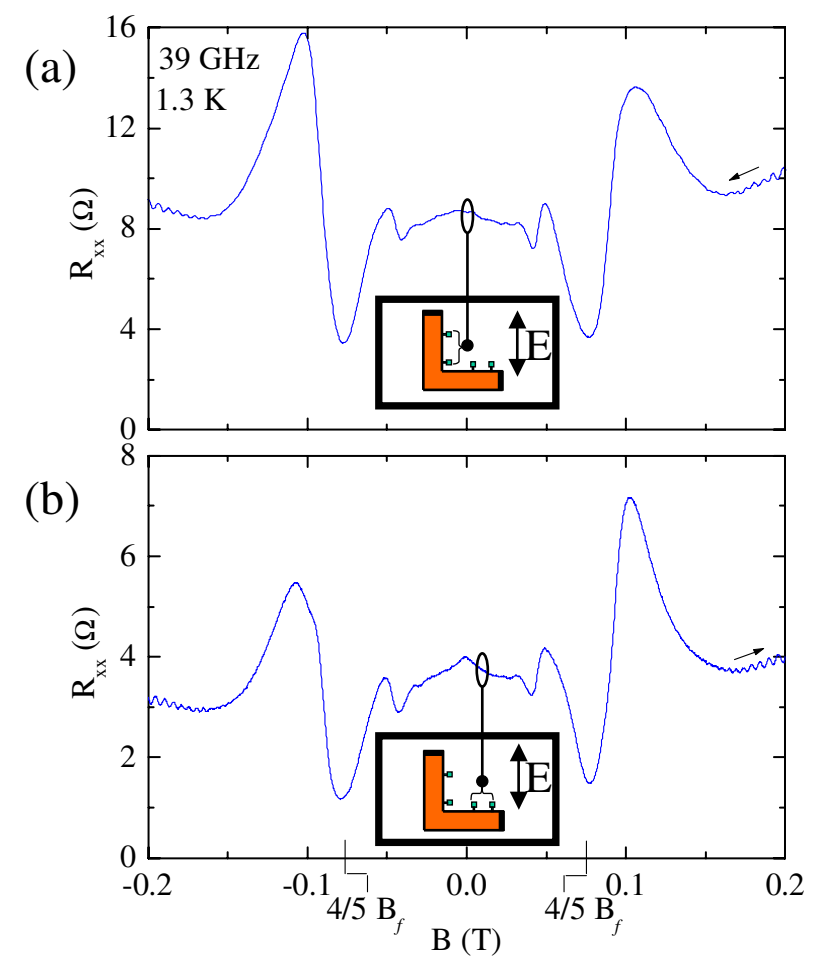

FIG. 7: Measurements carried out on an L-shaped sample mounted inside a WR-28 waveguide suggest insensitivity to the E-field polarization.(a) The current is oriented parallel to the E-field axis. (b) The current is oriented perpendicular to the E-field axis.

realizing zero-resistance in measurement.[19] A complementary theory by Shi and Xie,[13] also realized magnetoresistance oscillations.[6, 7, 8].

The observed zero-resistance states have also been related to a quantum Gunn effect,[21] while Rivera and Schulz have pointed out the possibility of gap formation

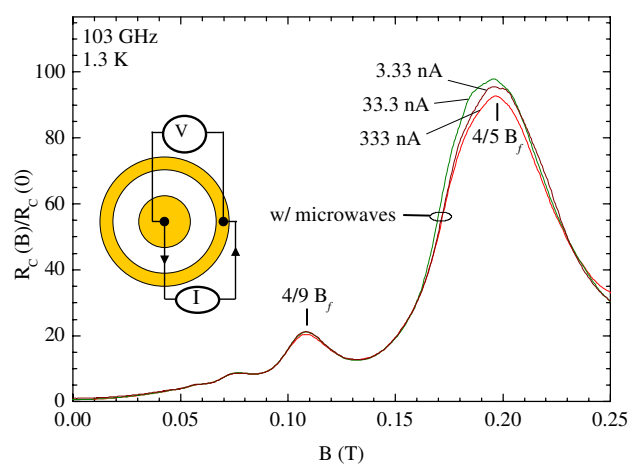

FIG. 8: In the Corbino geometry, resistance maxima occur in the vicinity of $B=[4 /(4 j+1)] B_{f}$, where $j=1,2,3, \ldots$ The Corbino resistance, $R_{C}$, was found to be independent of the current in the investigated regime. 


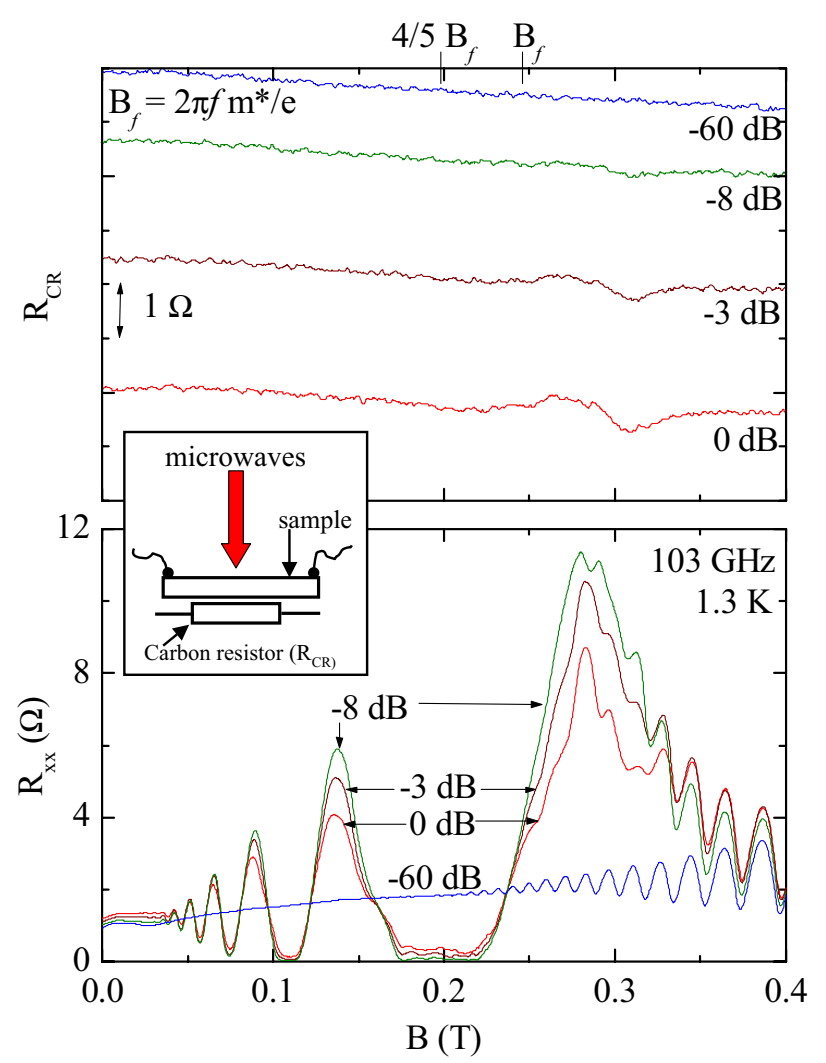

FIG. 9: These measurements examine the transmission characteristics of the 2DES under irradiation. Here, a carbon resistor placed below the sample served as the radiation detector. The detector resistance, $R_{C R}$, vs. $B$ (top). $R_{x x}$ vs. $B$ of the 2DES (bottom). This shows that when the power attenuation factor exceeds $-8 \mathrm{~dB}$, the oscillation amplitude decreases signifying "breakdown". The detector response suggests non monotonic transmission above $B_{f}$, while strong features do not occur below $B_{f}$ in top panel

due to the replication of Landau levels in the presence of radiation.[22] Although there has been progress in understanding aspects, see ref. [23, 24], many features including the activated temperature dependence, and the zero-resistance states themselves, could be better understood.

We acknowledge stimulating discussions with J. H. Smet, K. von Klitzing, V. Narayanamurti, and W. B. Johnson. The high quality MBE material was kindly provided by V. Umansky. Special thanks to K. von Klitzing for providing insight, support, and encouragement over many years.

* Electronic address: mani@deas.harvard.edu

[1] M. Tinkham, Introduction To Superconductivity, 2nd Edition, McGraw-Hill, New York, 1996.

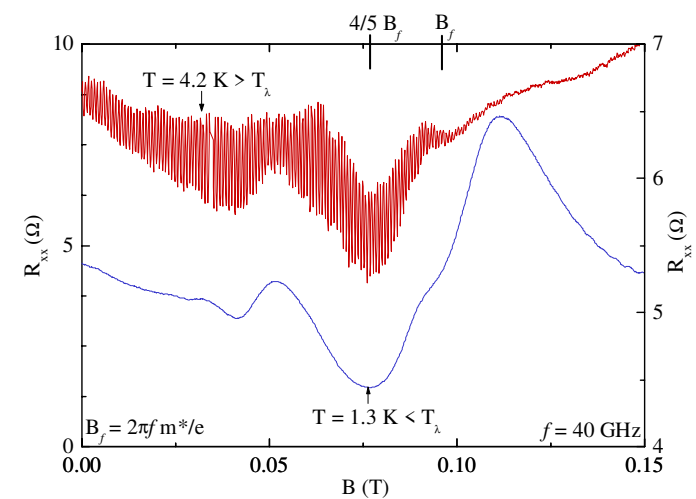

FIG. 10: Possible indication of energy absorption by the specimen below $B_{f}$. In a helium-4 (He-4) cryostat, a sample was mounted inside a waveguide, which was susceptible to He4 Thermo-Acoustic Oscillations(TAO). Above $\approx 2.2 \mathrm{~K}$, and below $B_{f}$, TAO's were observed, which produced sample vibrations, and therefore, electrical noise in the measurement. Below the Lambda-point, this noise producing mechanism vanished. The results suggest that energy absorption by the sample triggered the TAO's in this setup.

[2] R. E. Prange, S. M. Girvin (Eds.) The Quantum Hall Effect, 2nd edition, Springer, New York, 1990.

[3] T. Ando, A. B. Fowler, and F. Stern, Rev. Mod. Phys. 54(1982) 437.

[4] D. C. Tsui, H. L. Stormer, A. C. Gossard, Phys. Rev. B. 25 (1982) 1405 .

[5] R. G. Mani, J. H. Smet, K. von Klitzing, V. Narayanamurti, V. Umansky, Bull. Am. Phys. Soc. 46 (2001) 972.

[6] R. G. Mani, J. H. Smet, K. von Klitzing, V. Narayanamurti, W. B. Johnson, and V. Umansky, Nature 420 (2002) 646.

[7] R. G. Mani, J. H. Smet, K. von Klitzing, V. Narayanamurti, W. B. Johnson, V. Umansky, cond-mat/0303034.

[8] R. G. Mani, J. H. Smet, K. von Klitzing, V. Narayanamurti, W. B. Johnson, V. Umansky, cond-mat/0305507.

[9] R. G. Mani, et al., cond-mat/0306388.

[10] M. A. Zudov, R. R. Du, J. A. Simmons, J. L. Reno, Phys. Rev. B 64 (2001) 201311.

[11] P. D. Ye, et al., Appl. Phys. Lett. 79 (2001) 2193.

[12] A. C. Durst, S.Sachdev, N. Read, S. M. Girvin, condmat/0301569.

[13] J. Shi, X. C. Xie, cond-mat/0302393.

[14] A. A. Koulakov, M. E. Raikh, cond-mat/0302465.

[15] I. E. Spradley, S. W. K. Yuan, Adv. In Cryo. Eng., vol. 37A, Plenum Press, New York, 1992, p. 257.

[16] M. A. Zudov, R. R. Du, L. N. Pfeiffer, K. W. West, Phys. Rev. Lett. 90 (2003) 046807.

[17] J. C. Phillips, cond-mat/0212416; 0303184.

[18] V. I. Ryzhii, Sov. Phys. - Sol. St. 11 (1970) 2078.

[19] A. V. Andreev, I. L. Aleiner, A. J. Millis, condmat/0302063.

[20] P. W. Anderson, W. F. Brinkman, cond-mat/0302129.

[21] K. N. Shrivastava, cond-mat/0305032.

[22] P. H. Rivera, P. A. Schulz, cond-mat/0305019.

[23] R. Fitzgerald, Phys. Today 56 (4) (2003) 24.

[24] R. Klesse, F. Merz, cond-mat/0305492. 